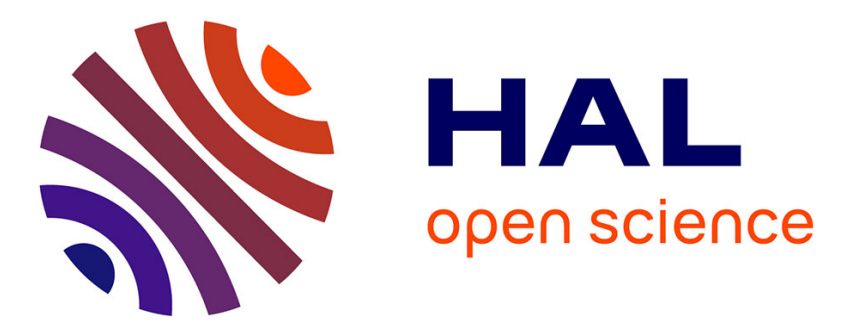

\title{
From local to global unmixing of hyperspectral images to reveal spectral variability
}

Guillaume Tochon, Lucas Drumetz, Miguel Angel Veganzones, Mauro Dalla Mura, Jocelyn Chanussot

\section{- To cite this version:}

Guillaume Tochon, Lucas Drumetz, Miguel Angel Veganzones, Mauro Dalla Mura, Jocelyn Chanussot. From local to global unmixing of hyperspectral images to reveal spectral variability. WHISPERS 2016 - 8th Workshop on Hyperspectral Image and Signal Processing: Evolution in Remote Sensing, Aug 2016, Los Angeles, CA, United States. hal-01356156

\section{HAL Id: hal-01356156 https://hal.science/hal-01356156}

Submitted on 25 Aug 2016

HAL is a multi-disciplinary open access archive for the deposit and dissemination of scientific research documents, whether they are published or not. The documents may come from teaching and research institutions in France or abroad, or from public or private research centers.
L'archive ouverte pluridisciplinaire HAL, est destinée au dépôt et à la diffusion de documents scientifiques de niveau recherche, publiés ou non, émanant des établissements d'enseignement et de recherche français ou étrangers, des laboratoires publics ou privés. 


\title{
FROM LOCAL TO GLOBAL UNMIXING OF HYPERSPECTRAL IMAGES TO REVEAL SPECTRAL VARIABILITY
}

 \\ ${ }^{1}$ GIPSA-lab, Grenoble Institute of Technology, Grenoble, France \\ ${ }^{2}$ Department of Electrical and Computer Engineering, University of Iceland, Reykjavik, Iceland
}

\begin{abstract}
The linear mixing model is widely assumed when unmixing hyperspectral images, but it cannot account for endmembers spectral variability. Thus, several workarounds have arisen in the hyperspectral unmixing literature, such as the extended linear mixing model (ELMM), which authorizes endmembers to vary pixelwise according to scaling factors, or local spectral unmixing (LSU) where the unmixing process is conducted locally within the image. In the latter case however, results are difficult to interpret at the whole image scale. In this work, we propose to analyze the local results of LSU within the ELMM framework, and show that it not only allows to reconstruct global endmembers and fractional abundances from the local ones, but it also gives access to the scaling factors advocated by the ELMM. Results obtained on a real hyperspectral image confirm the soundness of the proposed methodology.
\end{abstract}

Index Terms - Local spectral unmixing, extended linear mixing model, hyperspectral imagery

\section{INTRODUCTION}

Given a hyperspectral image (HSI), spectral unmixing is concerned with the identification of a set of signatures corresponding to pure macroscopic constituents, called endmembers and their respective proportions, termed fractional abundances, for all pixel spectra in the HSI. The linear mixing model (LMM) is a widely accepted assumption in which each pixel spectrum can be described as a linear combination of those endmembers and associated abundances [1].

The relative simplicity of the LMM has spawned numerous spectral unmixing techniques [2]. However, in spite of its popularity, the LMM suffers from several drawbacks. In real life scenarios, the mixing process cannot always be modeled as linear (due to multiple scattering or intimate mixing effects). Thus, nonlinear unmixing methods have been proposed (see [3] for a review). Moreover, the LMM implicitly assumes that each endmember can be properly represented by a single spectral signature, which is seldom true in practice due to the topography of the imaged scene and other physical aspects, including variable illumination conditions and the intrinsic variability of the materials (e.g., the amount of chlorophyll in vegetation). This endmember variability has received an increasing attention lately, and two main strategies have arisen in the literature [4]. The first one models the endmember variability through some particular multivariate probability distribution [5]. The second approach typically describes the spectral variability of a given endmember as a set of different instances of this endmember signature. Spectral bundles [6] are a typical example of such strategy where the spectral variability is not explicitly modeled. Lately, an extended linear mixing model (ELMM) has been proposed to address endmember variability while preserving the LMM framework [7], in which endmembers are allowed to vary from one pixel to another according to scaling factors.

Local spectral unmixing (LSU) is a potential solution to alleviate this spectral variability phenomenon. As a matter of fact, physical causes inducing spectral variability can be assumed to be sufficiently moderate locally in the image for the classical LMM to hold. In [8] for instance, the original image is first divided into several overlapping tiles, within which the spectral unmixing is locally conducted. To avoid blocking effects, another strategy was proposed in [9], where the LSU is performed on regions of a particular partition of the HSI. However, the main limitation of LSU techniques is that the induced endmembers and abundances are only defined locally in the image, and must somehow be post-processed to be interpretable at the whole image scale.

In this work, we propose to combine the LSU approach with the ELMM framework and show that it allows to derive global endmembers and abundances from their local counterparts, while providing additional information related to the spectral variability. The rest of this paper is organized as follow: section 2 presents more in details the ELMM framework [7] and a LSU approach relying on a hierarchical representation of the HSI [9]. Section 3 describes the proposed methodology to connect the previous two strategies in order to have access to global endmembers, abundances, as well as spectral variability. Conducted experiments and results are presented in section 4, while conclusion and perspectives are drawn in section 5 .

\section{SPECTRAL UNMIXING AND VARIABILITY}

\subsection{Extended Linear Mixing Model}

Let $\mathbf{X} \in \mathbb{R}^{N_{\lambda} \times N_{p}}$ be a HSI composed of $N_{p}$ pixels and comprising $N_{\lambda}$ spectral channels. Under the LMM assumption, each pixel spectrum $\mathbf{x} \in \mathbb{R}^{N_{\lambda}}$ can be written as a linear combination of $d$ endmember signatures $\mathbf{e}_{i}, i \in\{1, \ldots, d\}$ weighted by their corresponding fractional abundances $\phi_{\mathbf{x}, i}$, plus some additive noise $\boldsymbol{\eta}$ :

$$
\mathbf{x}=\sum_{i=1}^{d} \phi_{\mathbf{x}, i} \mathbf{e}_{i}+\boldsymbol{\eta},
$$

with the fractional abundances subject to positivity $\left(\phi_{\mathbf{x}, i} \geq 0 \forall i\right)$ and sum-to-one $\left(\sum_{i=1}^{d} \phi_{\mathbf{x}, i}=1\right)$ constraints. The geometrical interpretation of such model is that all data points $\mathbf{x}$ lie in a $(d-$ 1)-dimensional simplex whose $d$ vertices are the endmembers $\mathbf{e}_{i}$. In the ELMM model $[7,10]$ on the other hand, the endmember signatures $\mathbf{e}_{i}$ are no longer constrained to be fixed, but can vary from one pixel to another according to some non negative scaling factor $\lambda_{i}$ with respect to some reference signature $\mathbf{e}_{\mathbf{0}_{i}}$. Following, equation (1) rewrites:

$$
\mathbf{x}=\sum_{i=1}^{d} \phi_{\mathbf{x}, i} \lambda_{i} \mathbf{e}_{\mathbf{0}}+\boldsymbol{\eta} .
$$


As described in [10], equation (2) states that each pixel lies on a simplex given by a cross-section of the convex hypercone spanned by the reference endmembers $\mathbf{e}_{\mathbf{o}_{i}}$, and those parameters are estimated by solving an optimization procedure for all pixels of the HSI. The physical rationale behind the ELMM is to model variability due to illumination effects, but it also allows to recover information related to intrinsic variability [10].

In any case, the quality of the unmixing process for a given pixel is frequently evaluated by the root mean square error (RMSE) between the true pixel spectrum $\mathrm{x}$ and the one reconstructed using the induced endmembers and abundances $\hat{\mathbf{x}}$ :

$$
\epsilon(\mathbf{x}, \hat{\mathbf{x}})=\frac{1}{\sqrt{N_{\lambda}}}\|\mathbf{x}-\hat{\mathbf{x}}\|_{2} .
$$

\subsection{Local Spectral Unmixing}

In the LSU strategy, the spatial support $\operatorname{sp}(\mathbf{X})$ of the HSI $\mathbf{X}$ is first divided in a set of regions $\{\mathcal{R} \subseteq \operatorname{sp}(\mathbf{X})\}$ which may overlap or not, but such that the LMM can be assumed to hold in each region $\mathcal{R}$. The unmixing of a pixel $\mathbf{x}$ belonging to a particular region $\mathcal{R}$ is then performed using only the information contained in this region:

$$
\mathbf{x}=\sum_{i=1}^{d_{\mathcal{R}}} \phi_{\mathbf{x}, i}^{\mathcal{R}} \mathbf{e}_{i}^{\mathcal{R}}+\boldsymbol{\eta}
$$

where the $d_{\mathcal{R}}$ endmembers $\mathbf{e}_{i}^{\mathcal{R}}$ and their associated fractional abundances $\phi_{\mathbf{x}, i}^{\mathcal{R}}$ have been induced using only the spectral information contained in region $\mathcal{R}$.

The major challenge of LSU approaches concerns the definition of a suited set of regions $\{\mathcal{R}\}$. In this paper, we follow the work presented in [9] that makes use of a partition of the spatial support $\operatorname{sp}(\mathbf{X})$, $\pi=\left\{\mathcal{R}_{i} \subseteq \operatorname{sp}(\mathbf{X})\right\}$ such that $\operatorname{sp}(\mathbf{X})=\bigcup_{i} \mathcal{R}_{i}$ and $\mathcal{R}_{i} \cap \mathcal{R}_{j \neq i}=\emptyset$ extracted from a hierarchical representation of this HSI. More specifically, the HSI is first decomposed in a set of regions organized hierarchically (such that any two regions of this set are either disjoint or nested). The main advantage of hierarchical image decompositions is that they define regions at various scales ranging from very small (close to the pixels) to very large (up to the whole image spatial support) regions. The selection of regions with ideal scale to compose the resulting partition can then be formulated as an optimization problem conducted on the hierarchical structure [11]. Here, we exploit the idea presented in [12], where LSU is first performed over all regions of the hierarchical representation, and its performance is assessed by the local reconstruction errors of the regions. The final obtained partition of the HSI is such that its overall reconstruction error is minimal with respect to all the other possible partitions contained in the hierarchical representation.

\section{PROPOSED METHODOLOGY}

As explained in the previous section 2.2, the starting point of the proposed methodology is a partition $\pi=\left\{\mathcal{R}_{i}\right\}$ of the spatial support of the HSI, where LSU procedures have been conducted on each region $\mathcal{R}_{i}$ of the partition. Following [12], those regions have been designed to have minimal reconstruction errors. Thus, $d_{\mathcal{R}_{i}}$ local endmembers $\mathbf{e}_{1}^{\mathcal{R}_{i}}, \ldots, \mathbf{e}_{d_{\mathcal{R}_{i}}}^{\mathcal{R}_{i}}$ are available in each region $\mathcal{R}_{i}$, as well as their associated local fractional abundances $\phi_{x}^{\mathcal{R}_{i}}=\left[\phi_{\mathbf{x}, 1}^{\mathcal{R}_{i}}, \ldots, \phi_{\mathbf{x}, d_{\mathcal{R}_{i}}}^{\mathcal{R}_{i}}\right]$ for all pixel spectra $\mathrm{x}$ belonging to $\mathcal{R}_{i}$.

\subsection{Endmember clustering}

In a first step, all local endmembers are pooled together in a common set $\mathcal{E}_{\pi}=\left\{\mathbf{e}_{i}^{\mathcal{R} \in \pi}\right\}_{i=1}^{d_{\pi}}$ with $d_{\pi}=\sum_{\mathcal{R} \in \pi} d_{\mathcal{R}}$ being the total number of local endmembers that have been generated by the LSU approach. Then, this set $\mathcal{E}_{\pi}$ is clustered into $K$ clusters $\mathcal{C}_{1}, \ldots \mathcal{C}_{K}$ by means of some clustering algorithm, with cluster $\mathcal{C}_{k}=\left\{\mathbf{e}_{k, 1}^{\mathcal{R}}, \ldots \mathbf{e}_{k, d_{\mathcal{C}_{k}}}^{\mathcal{R}}\right\}$ being composed of $d_{\mathcal{C}_{k}}$ local endmembers, originating from various regions $\mathcal{R}$ of the partition $\pi$. Finally, the centroid $\mathbf{c}_{k}$ of each cluster $\mathcal{C}_{k}$ is retrieved

$$
\mathbf{c}_{k}=\frac{1}{d_{\mathcal{C}_{k}}} \sum_{i=1}^{d_{\mathcal{C}_{k}}} \mathbf{e}_{k, i}^{\mathcal{R}},
$$

and defined as the global endmember representing the $k^{\text {th }}$ cluster $\mathcal{C}_{k}$. As opposed to all local endmembers $\mathbf{e}_{k, i}$ belonging to this cluster, the centroid $\mathbf{c}_{k}$ is expected to properly describe the macroscopic material associated with cluster $\mathcal{C}_{k}$ across the whole image.

\subsection{Global abundance retrieval}

Once the global endmembers $\mathbf{c}_{1}, \ldots, \mathbf{c}_{K}$ have been defined, their associated global fractional abundances must be retrieved for all pixels spectra of the HSI. In particular, let $\mathbf{x}$ be such a pixel spectrum contained in region $\mathcal{R}$ of partition $\pi$, and let $\left[\phi_{\mathbf{x}, 1}^{\mathcal{R}}, \ldots, \phi_{\mathbf{x}, d_{\mathcal{R}}}^{\mathcal{R}}\right]$ and $\left[\psi_{\mathbf{x}, 1}, \ldots, \psi_{\mathbf{x}, K}\right]$ be its local and global fractional abundances, respectively. Then, three possible cases may occur:

- No local endmember $\mathbf{e}_{i}^{\mathcal{R}}$ has been clustered in $\mathcal{C}_{k}$. Thus, the $k^{\text {th }}$ macroscopic material represented by $\mathcal{C}_{k}$ is not contained in $\mathbf{x}$ and $\psi_{\mathbf{x}, k}=0$.

- There is a single local endmember $\mathbf{e}_{i_{0}}^{\mathcal{R}}$ belonging to cluster $\mathcal{C}_{k}$. Therefore, the proportion of this material shall not change within $\mathbf{x}$, hence $\psi_{\mathbf{x}, k}=\phi_{\mathbf{x}, i_{0}}^{\mathcal{R}}$.

- There are several local endmembers $\mathbf{e}_{i_{n}}^{\mathcal{R}}, n=1, \ldots, p$ grouped in the same cluster $\mathcal{C}_{k}$. In such situation, the material is locally variable within the region $\mathcal{R}$ (healthy and burnt grass for instance), but all contributions sum up with respect to the global instance of the material (being grass in the previous example). Thus, $\psi_{\mathbf{x}, k}=\sum_{n=1}^{p} \phi_{\mathbf{x}, i_{n}}^{\mathcal{R}}$.

All previous cases can be summarized as follows:

$$
\psi_{\mathbf{x}, k}=\sum_{i=1}^{d_{\mathcal{R}}} \mathbb{1}_{\left\{\mathbf{e}_{i}^{\mathcal{R}} \in \mathcal{C}_{k}\right\}} \phi_{\mathbf{x}, i}^{\mathcal{R}},
$$

where $\mathbb{1}_{\left\{\mathbf{e}_{i}^{\mathcal{R}} \in \mathcal{C}_{k}\right\}}=1$ if $\mathbf{e}_{i}^{\mathcal{R}} \in \mathcal{C}_{k}$ and 0 otherwise. Doing so for all pixels $\mathbf{x}$ of the HSI allows to reconstruct global fractional abundance maps.

\subsection{Estimation of spectral variability}

In the ELMM framework, each endmember $\mathbf{e}_{i}$ is authorized to vary pixelwise with respect to some reference endmember $\mathbf{e}_{\mathbf{0}_{i}}$ according to some local scaling factor $\lambda_{i}$, as described by equation (2). Here, we take advantage of this idea by considering cluster centroids $\mathbf{c}_{k}$ to be those reference endmembers and modeling all local endmembers belonging to this cluster $\mathcal{C}_{k}$ as some scaled versions of $\mathbf{c}_{k}$ :

$$
\mathbf{e}_{i}^{\mathcal{R}} \in \mathcal{C}_{k} \Rightarrow \mathbf{e}_{i}^{\mathcal{R}}=\lambda_{i} \mathbf{c}_{k}
$$

The scaling factor $\lambda_{i}$ associated with the local endmember $\mathbf{e}_{i}^{\mathcal{R}}$ can be recovered in practice by least square regression between $\mathbf{e}_{i}^{\mathcal{R}}$ and 
the centroid $\mathbf{c}_{k}$ of cluster $\mathcal{C}_{k}$ it belongs to:

$$
\lambda_{i}=\left(\mathbf{c}_{k}^{T} \mathbf{c}_{k}\right)^{-1} \mathbf{c}_{k}^{T} \mathbf{e}_{i}^{\mathcal{R}}
$$

with $(\cdot)^{T}$ being the transpose operator. Besides, equation (8) guarantees the local scaling factor $\lambda_{i}$ to be non negative.

Plugging equation (7) into equation (4) yields

$$
\mathbf{x}=\sum_{i=1}^{d_{\mathcal{R}}} \phi_{\mathbf{x}, i}^{\mathcal{R}} \lambda_{i} \mathbf{c}_{k_{i}}+\boldsymbol{\eta}
$$

where $k_{i} \in\{1, \ldots, K\}$ is the index of cluster $\mathbf{e}_{i}^{\mathcal{R}}$ belongs to. Equation (9) can be rewritten as

$$
\mathbf{x}=\sum_{k=1}^{K}\left(\sum_{i=1}^{d_{\mathcal{R}}} \phi_{\mathbf{x}, i}^{\mathcal{R}} \lambda_{i} \mathbb{1}_{\left\{\mathbf{e}_{i}^{\mathcal{R}} \in \mathcal{C}_{k}\right\}}\right) \mathbf{c}_{k}+\boldsymbol{\eta} .
$$

On the other hand, pixel $\mathrm{x}$ can also be decomposed with respect to the global ELMM framework as described by equation (2), namely

$$
\begin{aligned}
\mathbf{x} & =\sum_{k=1}^{K} \psi_{\mathbf{x}, k} \rho_{k} \mathbf{c}_{k}+\boldsymbol{\eta} \\
& =\sum_{k=1}^{K}\left(\sum_{i=1}^{d_{\mathcal{R}}} \phi_{\mathbf{x}, i}^{\mathcal{R}} \mathbb{1}_{\left\{\mathbf{e}_{i}^{\mathcal{R}} \in \mathcal{C}_{k}\right\}}\right) \rho_{k} \mathbf{c}_{k}+\boldsymbol{\eta}
\end{aligned}
$$

with $\rho_{k}$ being the global scaling factor associated with centroid $\mathbf{c}_{k}$. Hence, it is possible to estimate the global scaling factor $\rho_{k}$ for pixel $\mathbf{x}$ as a weighted average of its local scaling factors $\lambda_{i}$ and local abundances $\phi_{\mathbf{x}, i}^{\mathcal{R}}$ :

$$
\rho_{k}=\frac{\sum_{i=1}^{d_{\mathcal{R}}} \phi_{\mathbf{x}, i}^{\mathcal{R}} \lambda_{i} \mathbb{1}_{\left\{\mathbf{e}_{i}^{\mathcal{R}} \in \mathcal{C}_{k}\right\}}}{\sum_{i=1}^{d_{\mathcal{R}}} \phi_{\mathbf{x}, i}^{\mathcal{R}} \mathbb{1}_{\left\{\mathbf{e}_{i}^{\mathcal{R}} \in \mathcal{C}_{k}\right\}}}
$$

Note that, in the case where there is a single local endmember $\mathbf{e}_{i_{0}}^{\mathcal{R}}$ belonging to cluster $\mathcal{C}_{k}$, then $\rho_{k}=\lambda_{i_{0}}$ is constant for all pixels $\mathbf{x}$ of region $\mathcal{R}$. As a matter of fact, all pixels in $\mathcal{R}$ appear spatially homogeneous with respect to the material represented by $\mathcal{C}_{k}$. If there are at least two local endmembers belong to the same cluster $\mathcal{C}_{k}$ on the other hand, then the global scaling factor $\rho_{k}$ varies pixelwise. Finally, in the case where $\psi_{\mathbf{x}, \mathbf{k}}=\sum_{i=1}^{d_{\mathcal{R}}} \phi_{\mathbf{x}, i}^{\mathcal{R}} \mathbb{1}_{\left\{\mathbf{e}_{i}^{\mathcal{R}} \in \mathcal{C}_{k}\right\}}=0$ (that is, if pixel $\mathbf{x}$ does not contain the $k^{\text {th }}$ material), then $\rho_{k}$ is set to 1 .

\section{EXPERIMENTS}

\subsection{Experimental methodology}

We apply the proposed methodology to the HSI acquired over the campus of the University of Houston in 2012 and described in [13]. This image is composed of $340 \times 320$ pixels in spatial dimension, and comprises 144 bands. The study site features an urban area with a stadium, buildings, parking lots and roads, and some portions of grass and trees. A color composition of this HSI is presented by figure 1a. The partition $\pi$, input of the proposed methodology, is obtained following the procedure described in [12]. First a spatial pre-processing of the HSI is conducted in order to mitigate the effects of potential outliers [14]. Then, a binary partition tree (BPT) representation [15] is constructed using the spectral region model proposed in [12] (modeling all regions by their local endmembers) and the endmember-based distance as merging criterion [16]. A LSU procedure is conducted

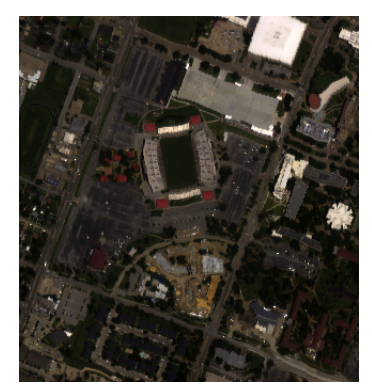

(a)

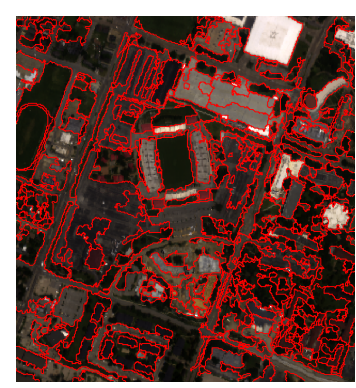

(b)
Fig. 1: (a) RGB composition of the Houston hyperspectral data set, and (b) resulting segmentation composed of 396 regions.

over each region of the BPT. The local intrinsic dimensionality $d_{\mathcal{R}}$ of each region is estimated using random matrix theory [17], as it proved to be reliable when working over small regions [18]. Local endmembers are estimated using the vertex component analysis (VCA) algorithm [19] and their fractional abundances are retrieved by non-negative sparse regression using a fractional semi-norm [20]. The partition $\pi$ extracted from the BPT structure is displayed by figure $1 \mathrm{~b}$ and composed of 396 regions. It achieves a trade-off between low region-wise maximal reconstruction errors (penalizing large regions with potential high reconstruction errors, which may be caused by the invalidity of the LMM within the region) and simplicity (penalizing partitions with too many regions) (see [12] for practical details).

All generated local endmembers are grouped in the set $\mathcal{E}_{\pi}$, eventually composed of 2957 individuals. This set $\mathcal{E}_{\pi}$ is divided into $K=12$ clusters following a multivariate Gaussian mixing model hypothesis, by application of the Expectation-Maximization algorithm [21]. Note that the total number of clusters $K$ has been set empirically. According to the proposed methodology, all cluster centroids $\mathbf{c}_{k}, k=1, \ldots, K$ are defined to be the ELMM reference endmembers. The associated global abundances $\psi_{\mathbf{x}, k}$ and global scaling factors $\rho_{k}$ are retrieved for all pixel spectra $\mathbf{x}$ in the HSI following the procedures exposed in section 3.2 and section 3.3, respectively. In order to evaluate the performance of the proposed methodology, we also unmix the image following the classical LMM scenario: $\mathrm{K}$ "classical" endmembers and associated fractional abundances are globally induced over the image, using the same set-up as the LSU (namely VCA for the endmember induction and non-negative sparse regression using fractional semi-norm for the abundances retrieval). In both cases, the quality of the unmixing for pixel spectrum $\mathbf{x}$ is evaluated by its reconstruction error (equation (3)).

\subsection{Results}

Figure 2 presents the obtained results for the semantic classes Asphalt, Vegetation and Metallic roofs. The first row of figure 2 displays the clusters obtained by the proposed strategy, where each blue spectrum depicts a local endmember obtained by LSU, the red spectrum is the cluster centroid, and the black spectrum is the corresponding endmember induced using the classical global approach. The second and third rows of figure 2 exhibit the fractional abundance maps for the classical global approach, and the proposed approach, respectively (with scales ranging from 0 (blue) to 1 (red). Finally, the bottom row displays the global scaling factors obtained by the proposed approach. Their values range from 0.5 (in blue) to 1.5 (in red). As remarked in [22], scaling factors are only relevant if the associated fractional abundances are high enough (greater than 0.3 in practice). In the opposite situation, the contribution of the associated endmembers to 

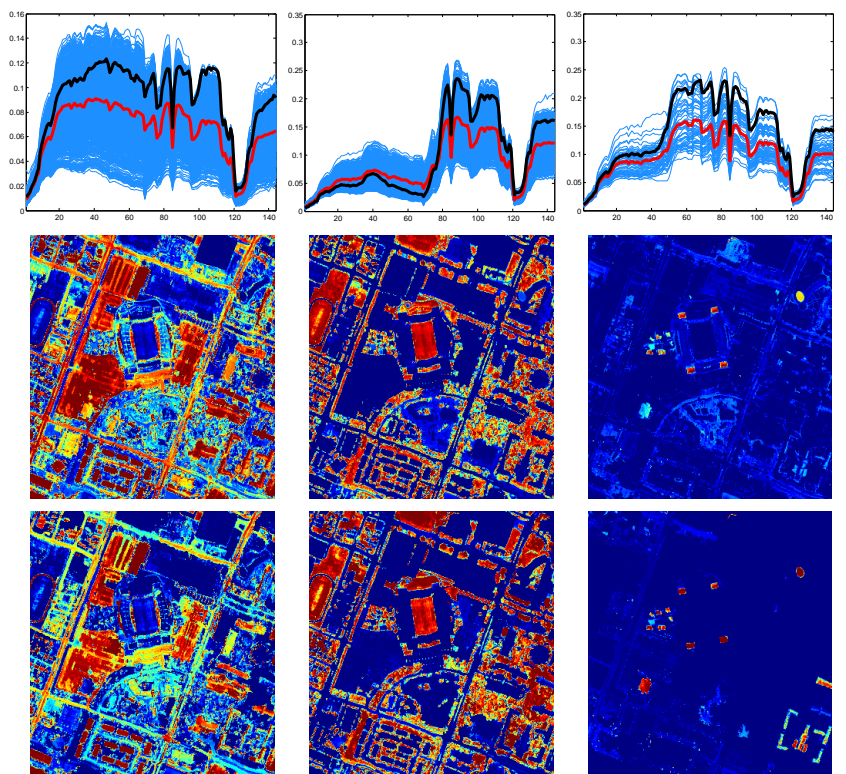

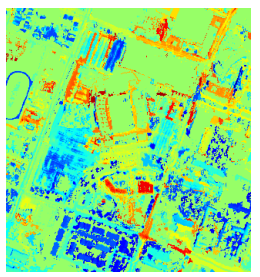

Asphalt

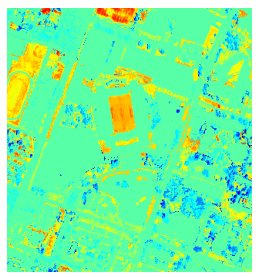

Vegetation



Metallic roofs
Fig. 2: First row: obtained clusters (in blue) along with their centroids (in red) and the classical global endmembers (in black). Second and third rows: fractional abundance maps associated to the global endmembers and the cluster centroids, respectively. Bottom row: scaling factor maps obtained by the proposed methodology.

the pixel spectra cannot be considered significant enough to reliably estimate their variability. In such case, scaling factors have been rounded to 1 for visualization purposes.

As it can be seen on the top row, the obtained clusters are spectrally coherent in the sense that all local endmembers grouped in the same cluster differ only from a scaling factor, which empirically validates the ELMM base assumption. Cluster centroids and classical global endmembers are also similar, up to some scaling factor, which confirms that the former can indeed be considered as global endmember instances. Nevertheless, while the obtained fractional abundance maps appear comparable for the Asphalt and Vegetation classes, which are well present across the image, it is different for the scarce Metallic roofs class. Several metallic roof endmembers have been extracted thanks to the LSU approach and clustered together, allowing to retrieve a clean abundance map, while the global approach leads to an abundance map where other structures are visible. Finally, the obtained scaling factor maps appear visually consistent, as the observed variations can be linked to the different shades within the parking lots for the Asphalt class for instance, or to the topography of the scene, as it is the case for Vegetation class (where it is possible to distinguish between trees with low scaling factors, and grass with higher scaling factors). It is even clearer when looking at the scaling factors associated to the Metallic roof class, as shown in figure 3. While the abundances of the classical global approach show some
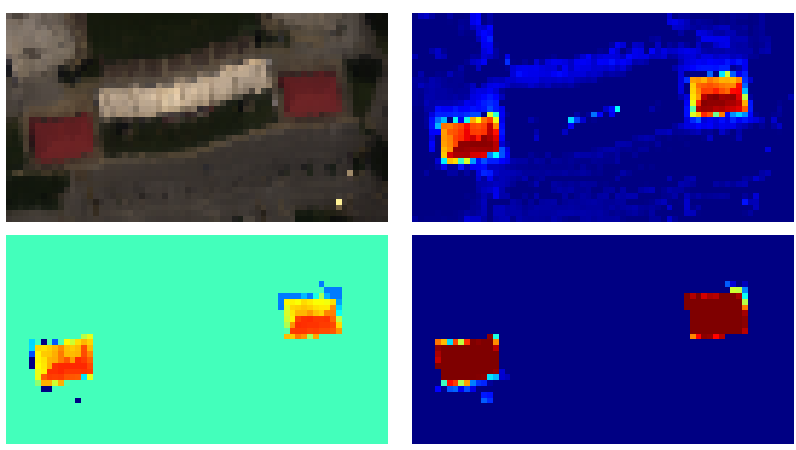

Fig. 3: Top row: crop of the RGB image, and global abundances associated to the Metallic roof class. Bottom row: obtained scaling factors and abundances for the Metallic roof class.

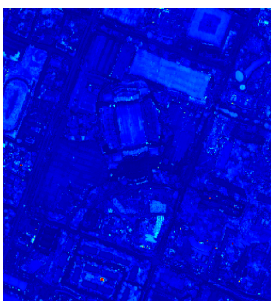

(a)



(b)

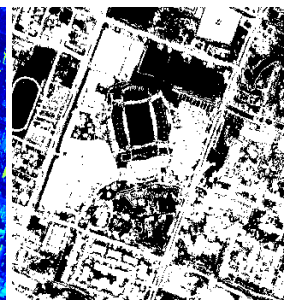

(c)
Fig. 4: Reconstruction map for (a) the proposed approach and (b) the classical global approach (the scale is saturated between 0 (blue) and 0.1 (red)), and (c) binary comparison of the two.

variability due to the topography of the two red roofs, this variability is clearly supported in our approach by the scaling factors, while the abundances remain relatively pure.

Finally, figure 4 displays the reconstruction error maps of the proposed (figure 4a) and classical global (figure 4b) approaches. As demonstrated by figure $4 \mathrm{c}$, the proposed approach globally yields lower reconstruction errors (all white pixels in figure $4 \mathrm{c}$ ) than the classical approach. It confirms that processing LSU results within the ELMM framework allows to take advantage of both the local validity of the LMM and the global variability of endmembers to better model the hyperspectral data set.

\section{CONCLUSION}

We presented here a novel methodology to interpret LSU results within the framework defined by the ELMM. By clustering all local endmembers provided by the LSU, and defining all cluster centroids to be the ELMM reference endmembers, we showed that is was not only possible to retrieve their associated global fractional abundances, but also their scaling factors advocated by the ELMM and accounting for the endmember spectral variability (which can be due to topographic or illumination effects). The proposed method was applied to a real hyperspectral data set, and the obtained results confirmed the soundness of combining the LSU to the ELMM framework.

Future work will be devoted to a exhaustive analysis of the proposed methodology over synthetic data sets, where is it possible to manually control the scaling factors and thus quantitatively assess the performance of the method. Sensitivity to the crucial choice of a clustering method will also be investigated. 


\section{REFERENCES}

[1] N. Keshava and J.F. Mustard, "Spectral unmixing," Signal Processing Magazine, IEEE, vol. 19, no. 1, pp. 44-57, 2002.

[2] J.M. Bioucas-Dias, A. Plaza, N. Dobigeon, M. Parente, Q Du, P. Gader, and J. Chanussot, "Hyperspectral unmixing overview: Geometrical, statistical, and sparse regression-based approaches," Selected Topics in Applied Earth Observations and Remote Sensing, IEEE Journal of, vol. 5, no. 2, pp. 354379, 2012.

[3] R. Heylen, M. Parente, and P. Gader, "A review of nonlinear hyperspectral unmixing methods," Selected Topics in Applied Earth Observations and Remote Sensing, IEEE Journal of, vol. 7, no. 6, pp. 1844-1868, 2014.

[4] A. Zare and K.C. Ho, "Endmember variability in hyperspectral analysis: Addressing spectral variability during spectral unmixing," Signal Processing Magazine, IEEE, vol. 31, no. 1, pp. 95-104, 2014.

[5] X. Du, A. Zare, P. Gader, and D. Dranishnikov, "Spatial and spectral unmixing using the beta compositional model," $\mathrm{Se}$ lected Topics in Applied Earth Observations and Remote Sensing, IEEE Journal of, vol. 7, no. 6, pp. 1994-2003, 2014.

[6] B. Somers, M. Zortea, A. Plaza, and G. P. Asner, "Automated extraction of image-based endmember bundles for improved spectral unmixing," Selected Topics in Applied Earth Observations and Remote Sensing, IEEE Journal of, vol. 5, no. 2, pp. 396-408, 2012.

[7] M. A. Veganzones, L. Drumetz, G. Tochon, M. Dalla Mura, A. Plaza, J. M. Bioucas-Dias, and J. Chanussot, "A new extended linear mixing model to address spectral variability," in IEEE Workshop on Hyperspectral Image and Signal Processing: Evolution in Remote Sensing (WHISPERS 2014), 2014, pp. 1-4.

[8] K. Canham, A. Schlamm, A. Ziemann, B. Basener, and D. Messinger, "Spatially adaptive hyperspectral unmixing," Geoscience and Remote Sensing, IEEE Transactions on, vol. 49, no. 11, pp. 4248-4262, 2011.

[9] L. Drumetz, M. A. Veganzones, R. Marrero, G. Tochon, M. Dalla Mura, A. Plaza, and J. Chanussot, "Binary partition tree-based local spectral unmixing," in IEEE Workshop on Hyperspectral Image and Signal Processing: Evolution in Remote Sensing (WHISPERS 2014), 2014, pp. 1-4.

[10] L. Drumetz, S. Henrot, M. A. Veganzones, J. Chanussot, and C. Jutten, "Blind hyperspectral unmixing using an extended linear mixing model to address spectral variability," in IEEE Workshop on Hyperspectral Image and Signal Processing: Evolution in Remote Sensing (WHISPERS 2015), 2015, pp. 1-4.

[11] Y. Tarabalka, J. C. Tilton, J. A. Benediktsson, and J. Chanussot, "A marker-based approach for the automated selection of a single segmentation from a hierarchical set of image segmentations," Selected Topics in Applied Earth Observations and Remote Sensing, IEEE Journal of, vol. 5, no. 1, pp. 262-272, 2012.

[12] M.A. Veganzones, G. Tochon, M. Dalla-Mura, A.J. Plaza, and J. Chanussot, "Hyperspectral image segmentation using a new spectral unmixing-based binary partition tree representation," Image Processing, IEEE Transactions on, vol. 23, no. 8, pp. 3574-3589, 2014.

[13] C. Debes, A. Merentitis, R. Heremans, J. Hahn, N. Frangiadakis, T. van Kasteren, W. Liao, R. Bellens, A. Pizurica, S. Gautama, et al., "Hyperspectral and LiDAR data fusion: Outcome of the 2013 GRSS data fusion contest," Selected Topics in Applied Earth Observations and Remote Sensing, IEEE Journal of, vol. 7, no. 6, pp. 2405-2418, 2014.

[14] M. Zortea and A. J. Plaza, "Spatial preprocessing for endmember extraction," Geoscience and Remote Sensing, IEEE Transactions on, vol. 47, no. 8, pp. 2679-2693, 2009.

[15] S. Valero, P. Salembier, and J. Chanussot, "Hyperspectral image representation and processing with binary partition trees," Image Processing, IEEE Transactions on, vol. 22, no. 4, pp. 1430-1443, 2013.

[16] M. Grana and M. A. Veganzones, “An endmember-based distance for content based hyperspectral image retrieval," Pattern Recognition, vol. 45, no. 9, pp. 3472-3489, 2012.

[17] K. Cawse-Nicholson, S. B. Damelin, A. Robin, and M. Sears, "Determining the intrinsic dimension of a hyperspectral image using random matrix theory," Image Processing, IEEE Transactions on, vol. 22, no. 4, pp. 1301-1310, 2013.

[18] L. Drumetz, M. A. Veganzones, R. Marrero Gmez, G. Tochon, M. Dalla Mura, G. A. Licciardi, C. Jutten, and J. Chanussot, "Hyperspectral local intrinsic dimensionality," IEEE Transactions on Geoscience and Remote Sensing, vol. PP, no. 99, pp. 1-16, 2016.

[19] J. M. B. Nascimento and J. M. Bioucas Dias, "Vertex component analysis: A fast algorithm to unmix hyperspectral data," Geoscience and Remote Sensing, IEEE Transactions on, vol. 43, no. 4, pp. 898-910, 2005.

[20] T. Meyer, L. Drumetz, J. Chanussot, A. L. Bertozzi, and C. Jutten, "Hyperspectral unmixing with material variability using social sparsity.," in IEEE International Conference on Image Processing, 2016, (submitted).

[21] Y. Tarabalka, J. A. Benediktsson, and J. Chanussot, "Spectralspatial classification of hyperspectral imagery based on partitional clustering techniques," Geoscience and Remote Sensing, IEEE Transactions on, vol. 47, no. 8, pp. 2973-2987, 2009.

[22] L Drumetz, M. A. Veganzones, S. Henrot, R. Phlypo, J. Chanussot, and C. Jutten, "Blind hyperspectral unmixing using an extended linear mixing model to address spectral variability," Image Processing, IEEE Transactions on, 2016, (submitted). 